\title{
Connecting various types of cyclic contractions and contractive self-mappings with Hardy-Rogers self-mappings
}

\author{
Panda Sumati Kumari ${ }^{1 *}$ and Dinesh Panthi ${ }^{2}$
}

${ }^{*}$ Correspondence:
mumy143143143@gmail.com
${ }^{1}$ Department of Mathematics,
National Institute of Technology,
Andhra Pradesh, AP, India
Full list of author information is
available at the end of the article

\begin{abstract}
In this paper, we introduce the new classes of Hardy-Rogers type cyclic contractions and prove pertinent fixed point theorems for these Hardy-Rogers type contractions in the generating space of a $b$-dislocated metric family.

MSC: $47 \mathrm{H} 10 ; 54 \mathrm{H} 25$

Keywords: modified Hardy-Rogers cyclic contraction; Hardy-Rogers cyclic orbital contraction; Hardy-Rogers F-contraction; generating space of $b$-dislocated metric family; b-dislocated metric; dislocated metric; fixed point
\end{abstract}

\section{Introduction and preliminaries}

Throughout this paper the characters $\mathbb{R}^{+}$and $\mathbb{N}$ represent the set of non-negative real numbers and the set of positive integer numbers, respectively.

It is well known that Banach's contraction mapping theorem is one of the pivotal results of metric fixed point theory.

Theorem 1.1 (Banach [1]) If $(X, d)$ is a complete metric space and $T: X \rightarrow X$ is a selfmapping such that

$$
d(T x, T y) \leq \alpha d(x, y)
$$

for all $x, y \in X$, where $0 \leq \alpha<1$, then $T$ has a unique fixed point.

Theorem 1.2 (Kannan [2]) If $(X, d)$ is a complete metric space and $T: X \rightarrow X$ is a selfmapping such that

$$
d(T x, T y) \leq \beta[d(x, T x)+d(y, T y)]
$$

for all $x, y \in X$, where $0 \leq \beta<\frac{1}{2}$, then $T$ has a unique fixed point.

Theorem 1.3 (Reich [3]) If $(X, d)$ is a complete metric space and $T: X \rightarrow X$ is a selfmapping such that

$$
d(T x, T y) \leq \alpha d(x, y)+\beta d(x, T x)+\gamma d(y, T y)
$$

\section{Springer}

(c) 2016 Kumari and Panthi. This article is distributed under the terms of the Creative Commons Attribution 4.0 International License (http://creativecommons.org/licenses/by/4.0/), which permits unrestricted use, distribution, and reproduction in any medium, provided you give appropriate credit to the original author(s) and the source, provide a link to the Creative Commons license, and indicate if changes were made. 
for all $x, y \in X$, where $\alpha, \beta, \gamma$ are non-negative constants with $\alpha+\beta+\gamma<1$, then $T$ has a unique fixed point.

Theorem 1.4 (Chatterjea [4]) If $(X, d)$ is a complete metric space and $T: X \rightarrow X$ is a selfmapping such that

$$
d(T x, T y) \leq \gamma[d(x, T y)+d(y, T x)]
$$

for all $x, y \in X$, where $0 \leq \gamma<\frac{1}{2}$, then $T$ has a unique fixed point.

Theorem 1.5 (Ćirić [5]) If $(X, d)$ is a complete metric space and $T: X \rightarrow X$ is a selfmapping such that

$$
d(T x, T y) \leq \alpha d(x, y)+\beta d(x, T x)+\gamma d(y, T y)+\delta[d(x, T y)+d(y, T x)]
$$

for all $x, y \in X$, where $\alpha, \beta, \gamma, \delta$ are non-negative constants with $\alpha+\beta+\gamma+2 \delta<1$, then $T$ has a unique fixed point.

Theorem 1.6 (Hardy and Rogers [6]) If $(X, d)$ is a complete metric space and $T: X \rightarrow X$ is a self-mapping such that

$$
d(T x, T y) \leq \alpha d(x, y)+\beta d(x, T x)+\gamma d(y, T y)+\delta d(x, T y)+\eta d(y, T x),
$$

for all $x, y \in X$, where $\alpha, \beta, \gamma, \delta, \eta$ are non-negative constants with $\alpha+\beta+\gamma+\delta+\eta<1$, then $T$ has a unique fixed point.

We note some important consequences as follows:

- The Banach fixed point theorem ensures the existence and uniqueness of fixed points of certain self-maps of metric spaces, and it gives a useful constructive method to find those fixed points.

- It is interesting that Kannan's fixed point theorem is very salient because the author of [7] proved that Kannan's theorem describes the completeness of the metric. More readily, a metric space $X$ is complete if and only if each and every Kannan mapping on $X$ has a fixed point. Moreover, the conditions (1) and (2) are independent, as was shown by two examples in [8].

- Reich generalized the Banach and Kannan fixed point theorems. An example in [3] indicates that the condition (3) is a qualified generalization of (1) and (2).

- A similar Kannan type of contractive condition has been studied by Chatterjea[4]. In Theorems 1.2 and 1.4, there is no necessity of continuity.

- A mapping satisfying (5) is called a generalized contraction.

- The importance of the Hardy-Rogers contraction is that this condition generalizes all the known named contractive conditions, but not conversely.

- Many generalizations of Hardy-Rogers theorem can be found in the literature. (See for example [9-11].)

In 2003, Kirk et al. [12] introduced cyclic contractions in metric spaces and investigated the existence of proximity points and fixed points in view of cyclic contraction mappings as follows. 
Definition 1.7 [12] Let $A$ and $B$ be non-empty subsets of a set $X$. A map $T: A \cup B \rightarrow A \cup B$ is said to be a cyclic map if $T(A) \subseteq B$ and $T(B) \subseteq A$.

Theorem 1.8 [12] Let $A$ and $B$ be non-empty subsets of a metric space $(X, d)$ and $T: A \cup$ $B \rightarrow A \cup B$ be a cyclic map. If there exists $k \in[0,1)$ such that

$$
d(T x, T y) \leq k(d(x, y))
$$

for all $x \in A$ and $y \in B$, then $T$ has a unique fixed point in $A \cap B$.

Since then, a huge number of authors continued the investigation and more results have been obtained, such as [13-19].

In 2010, Karpagam and Agrawal [20] introduced the notion of cyclic orbital contraction and proved a unique fixed point theorem for such a map.

Theorem 1.9 [20] Let $A$ and $B$ be non-empty closed subsets of a complete metric space $(X, d)$ and $T: A \cup B \rightarrow A \cup B$ be a cyclic orbital contraction; i.e., if for some $x \in A$ there exists a $k_{x} \in(0,1)$ such that

$$
d\left(T^{2 n} x, T y\right) \leq k_{x} d\left(T^{2 n-1} x, y\right)
$$

for all $n \in \mathbb{N}$ and $y \in A$, then $T$ has a unique fixed point in $A \cap B$.

Further, many results dealing with cyclic orbital contractions have appeared in the literature (see, e.g., [21, 22]).

In 2012, Wardowski [23] introduced a different type of contraction called an Fcontraction and proved a new fixed point theorem, which generalizes the Banach contraction principle in a new way with supporting examples.

Definition 1.10 [23] Let $F: \mathbb{R}^{+} \rightarrow \mathbb{R}$ be a mapping satisfying:

(i) $F$ is strictly increasing, i.e. for all $\alpha, \beta \in \mathbb{R}^{+}$such that $\alpha<\beta, F(\alpha)<F(\beta)$.

(ii) For each sequence $\left\{\alpha_{n}\right\}_{n \in \mathbb{N}}$ of positive numbers $\lim _{n \rightarrow \infty} \alpha_{n}=0$ iff $\lim _{n \rightarrow \infty} F\left(\alpha_{n}\right)=-\infty$.

(iii) There exists $k \in(0,1)$ such that $\lim _{\alpha \rightarrow 0^{+}} \alpha^{k} F(\alpha)=0$.

A mapping $T: X \rightarrow X$ is said to be an $F$-contraction if there exists $\tau>0$ such that, for all $x, y \in X, d(T x, T y)>0$, we have $\tau+F(d(T x, T y)) \leq F(d(x, y))$.

Theorem 1.11 [23] Let $(X, d)$ be a complete metric space and let $T: X \rightarrow X$ be an Fcontraction then $T$ has a unique fixed point $x^{*} \in X$ and for every $x_{0} \in X$ a sequence $\left\{T^{n} x_{0}\right\}_{n \in \mathbb{N}}$ is convergent to $x^{*}$.

Further, some results dealing with an F-contraction have appeared in the literature (see, e.g., $[24-26])$.

The standard metric space has been generalized in different ways: see for example,

(a) S-metric space by Sedghi et al. [27];

(b) Complex valued metric space by Azam et al. [28]; 
(c) A-metric space by Abbas et al. [29];

(d) Dislocated symmetric space by Sarma et al. [30];

(e) Quasi-symmetric space by Kumari et al. [31];

(f) Dislocated Uniform space by Kumari et al. [32].

Apart from above, very recently, Kumari and Panthi [33] introduced the concepts of 'generating space of a $b$-dislocated quasi-metric family' (abbreviated ' $G_{b d q}$-family'), 'generating space of $b$-dislocated metric family' (abbreviated ' $G_{b d}$-family'), and 'generating space of $b$ quasi-metric family' (abbreviated ' $G_{b q}$-family'). One also proved the existence of unique fixed point theorems in weaker forms of a generating spaces by using various cyclic contractive conditions.

Definition 1.12 [33] Let $X$ be a non-empty set and $\left\{d_{\alpha}: \alpha \in(0,1]\right\}$ a family of mappings $d_{\alpha}$ of $X \times X$ into $\mathbb{R}^{+}$. Then $\left(X, d_{\alpha}\right)$ is called a generating space of a $b$-dislocated metric family if it satisfies the following conditions for any $x, y, z \in X$ and $s \geq 1$.

(i) $d_{\alpha}(x, y)=0$ implies $x=y$.

(ii) $d_{\alpha}(x, y)=d_{\alpha}(y, x)$.

(iii) For any $\alpha \in(0,1]$ there exists $\beta \in(0, \alpha]$ such that $d_{\alpha}(x, z) \leq s\left[d_{\beta}(x, y)+d_{\beta}(y, z)\right]$;

(iv) For any $x, y \in X, d_{\alpha}(x, y)$ is non-increasing and left continuous in $\alpha$.

Definition 1.13 [33]

1. Let $\left(X, d_{\alpha}\right)$ be a $G_{b d}$-family and $\left\{x_{n}\right\}$ be a sequence in $X$. We say that $\left\{x_{n}\right\}$ $G_{b d}$-converges to $x$ in $\left(X, d_{\alpha}\right)$ if $\lim _{n \rightarrow \infty} d_{\alpha}\left(x_{n}, x\right)=0$ for all $\alpha \in(0,1]$.

In this case we write $x_{n} \rightarrow x$.

2. Let $\left(X, d_{\alpha}\right)$ be a $G_{b d}$-family and let $A \subseteq X, x \in X$. We say that $x$ is a $G_{b d}$-limit point of $A$ if there exists a sequence $\left\{x_{n}\right\}$ in $A-\{x\}$ such that $\lim _{n \rightarrow \infty} x_{n}=x$.

3. A sequence $\left\{x_{n}\right\}$ in a $G_{b d}$-family is called a $G_{b d}$-Cauchy sequence if, given $\epsilon>0$, there exists $n_{0} \in \mathbb{N}$ such that for all $n, m \geq n_{0}$, we have $d_{\alpha}\left(x_{n}, x_{m}\right)<\epsilon$ or $\lim _{n, m \rightarrow \infty} d_{\alpha}\left(x_{n}, x_{m}\right)=0$ for all $\alpha \in(0,1]$.

4. A $G_{b d}$-family $\left(X, d_{\alpha}\right)$ is called complete if every $G_{b d}$-Cauchy sequence in $X$ is $G_{b d}$-convergent.

A similar argument can be found in [34-36].

Remark 1.14 [33] Every $G_{b d}$-convergent sequence in a $G_{b d}$-family is $G_{b d}$-Cauchy.

Motivated by all above facts, we introduce the new classes of Hardy-Rogers type contractions and prove some fixed point theorems using various types of Hardy-Rogers contractions in the context of generating space of a $b$-dislocated metric family. Our main theorems extend and unify existing results in the recent literature. Then we present several examples to illustrate the theorems.

\section{Main results}

In this section, we derive some fixed point theorems with examples in the context of a generating space of a $b$-dislocated metric family.

Definition 2.1 Let $A$ and $B$ be non-empty subsets of a $G_{b d}$-family $\left(X, d_{\alpha}\right)$. A cyclic map $T: A \cup B \rightarrow A \cup B$ is said to be a modified Hardy-Rogers cyclic contraction if we have 


$$
\begin{aligned}
d_{\alpha}(T x, T y) \leq & \alpha d_{\alpha}(x, y)+\beta d_{\alpha}(x, T y)+\gamma d_{\alpha}(y, T x)+\delta d_{\alpha}(y, T y) \\
& +\eta \frac{d_{\alpha}(y, T y)\left[1+d_{\alpha}(x, T x)\right]}{1+d_{\alpha}(x, y)}+\lambda \frac{d_{\alpha}(y, T y)+d_{\alpha}(y, T x)}{1+d_{\alpha}(y, T y) d_{\alpha}(y, T x)} \\
& +\mu \frac{d_{\alpha}(x, T x)\left[1+d_{\alpha}(y, T x)\right]}{1+d_{\alpha}(x, y)+d_{\alpha}(y, T y)}
\end{aligned}
$$

where $\alpha, \beta, \gamma, \delta, \eta, \lambda, \mu \geq 0$ with $s \alpha+\left(s^{2}+s\right) \beta+2 s^{2} \gamma+\delta+\eta+\lambda+s \mu<1$ and for all $x, y \in X$.

Theorem 2.2 Let $\left(X, d_{\alpha}\right)$ be a complete $G_{b d}$-family, $A$ and $B$ be non-empty closed subsets of $X$. Let $T: A \cup B \rightarrow A \cup B$ be a continuous cyclic mapping satisfying the modified HardyRogers cyclic contraction. Then $T$ has a unique fixed point in $A \cap B$.

Proof Let $x_{0}$ be arbitrary in $X$; we define a sequence $\left\{x_{n}\right\}$ as $x_{0}, x_{1}=T x_{0}, x_{2}=T x_{1}, \ldots, x_{n+1}=$ $T x_{n}$ for all $n \in \mathbb{N}$.

$$
\begin{aligned}
d_{\alpha}\left(T x, T^{2} x\right)= & d_{\alpha}(T x, T(T x)) \\
\leq & \alpha d_{\alpha}(x, T x)+\beta d_{\alpha}\left(x, T^{2} x\right)+\gamma d_{\alpha}(T x, T x)+\delta d_{\alpha}\left(T x, T^{2} x\right) \\
& +\eta \frac{d_{\alpha}\left(T x, T^{2} x\right)\left[1+d_{\alpha}(x, T x)\right]}{1+d_{\alpha}(x, T x)}+\lambda \frac{d_{\alpha}\left(T x, T^{2} x\right)+d_{\alpha}(T x, T x)}{1+d_{\alpha}\left(T x, T^{2} x\right) d_{\alpha}(T x, T x)} \\
& +\mu \frac{d_{\alpha}(x, T x)\left[1+d_{\alpha}(T x, T x)\right]}{1+d_{\alpha}(x, T x)+d_{\alpha}\left(T x, T^{2} x\right)} \\
= & \alpha d_{\alpha}(x, T x)+s \beta\left[d_{\beta}(x, T x)+d_{\beta}\left(T x, T^{2} x\right)\right]+2 s \gamma d_{\beta}(x, T x)+\delta d_{\alpha}\left(T x, T^{2} x\right) \\
& +\eta d_{\alpha}\left(T x, T^{2} x\right)+\lambda d_{\alpha}\left(T x, T^{2} x\right)+\mu d_{\alpha}(x, T x) \\
= & (\alpha+s \beta+2 s \gamma+\mu) d_{\alpha}(x, T x),
\end{aligned}
$$

which implies

$$
(1-s \beta-\delta-\eta-\lambda) d_{\alpha}\left(T x, T^{2} x\right) \leq(\alpha+s \beta+2 s \gamma+\mu) d_{\alpha}(x, T x)
$$

Clearly, $d_{\alpha}\left(T x, T^{2} x\right) \leq h d_{\alpha}(x, T x)$.

Here $h=\frac{\alpha+s \beta+2 s \gamma+\mu}{1-s \beta-\delta-\eta-\lambda}<\frac{1}{s}<1$.

Similarly,

$$
d_{\alpha}\left(T^{2} x, T^{3} x\right) \leq h d_{\alpha}\left(T x, T^{2} x\right) \leq h^{2} d_{\alpha}(x, T x) .
$$

For all $n \in \mathbb{N}$, we get

$$
d_{\alpha}\left(T^{n} x, T^{n+1} x\right) \leq h^{n} d_{\alpha}(x, T x) .
$$

Let $n, m \in \mathbb{N}$ with $m>n$, by using the definition of $G_{b d}$-family, we have

$$
\begin{aligned}
d_{\alpha}\left(T^{n} x, T^{m} x\right) & \leq s\left[d_{\beta}\left(T^{n} x, T^{n+1} x\right)+d_{\beta}\left(T^{n+1} x, T^{m} x\right)\right] \\
& \leq s d_{\beta}\left(T^{n} x, T^{n+1} x\right)+s d_{\beta}\left(T^{n+1} x, T^{m} x\right) \\
& \leq s d_{\beta}\left(T^{n} x, T^{n+1} x\right)+s^{2} d_{\beta}\left(T^{n+1} x, T^{n+2} x\right)+s^{2} d_{\beta}\left(T^{n+2} x, T^{m} x\right) .
\end{aligned}
$$


By repeating this process we get

$$
\begin{aligned}
d_{\alpha}\left(T^{n} x, T^{m} x\right) & \leq s d_{\beta}\left(T^{n} x, T^{n+1} x\right)+s^{2} d_{\beta}\left(T^{n+1} x, T^{n+2} x\right)+s^{3} d_{\beta}\left(T^{n+2} x, T^{n+3} x\right)+\cdots \\
& \vdots \\
& \leq\left(s h^{n}+s^{2} h^{n+1}+s^{3} h^{n+2}+\cdots\right) d_{\alpha}(x, T x) \\
& =s h^{n}\left(1+s h+(s h)^{2}+\cdots\right) d_{\alpha}(x, T x) \\
& \leq\left(\frac{s h^{n}}{1-s h}\right) d_{\alpha}(x, T x) .
\end{aligned}
$$

By taking the limits as $m \rightarrow \infty$, we get $d_{\alpha}\left(T^{n} x, T^{m} x\right) \rightarrow 0$ for all $\alpha \in[0,1)$ as $h s<1$.

Hence $\left\{T^{n} x\right\}$ is a $G_{b d}$-Cauchy sequence.

Since $\left(X, d_{\alpha}\right)$ is $G_{b d}$-complete, we see that $\left\{T^{n} x\right\} G_{b d}$-converges to some $u \in X$ for all $\alpha \in[0,1)$. We note that $\left\{T^{2 n} x\right\}$ is a sequence in $A$ and $\left\{T^{2 n-1} x\right\}$ is a sequence in $B$ such a way that both sequences tend to the same limit $u$.

Since $A$ and $B$ are closed, we have $u \in A \cap B$, and then $A \cap B \neq \emptyset$.

Now, we will show that $T u=u$.

Consider

$$
d_{\alpha}\left(T^{n} x, T u\right) \leq s\left[d_{\beta}\left(T^{n} x, T^{n+1} x\right)+d_{\beta}\left(T^{n+1} x, T u\right)\right]
$$

Since $T$ is continuous, $\lim _{n \rightarrow \infty} d_{\beta}\left(T^{n+1} x, T u\right)=0$ and $d_{\beta}\left(T^{n} x, T^{n+1} x\right) \leq h^{n} d_{\beta}(x, T x)$.

This implies

$$
d_{\beta}\left(T^{n} x, T^{n+1} x\right) \leq \operatorname{sh}^{n} d_{\beta}(x, T x) \leq(s h)^{n} d_{\beta}(x, T x) .
$$

Letting $n \rightarrow \infty$ in the above inequality (8), we get

$$
d_{\alpha}(u, T u)=0, \quad \text { since } s h<1 .
$$

Thus $u=T u$. Hence $u$ is a fixed point of $T$.

Finally, to obtain the uniqueness of a fixed point, let $v \in X$ be another fixed point of $T$ such that $T v=v$.

Then we have

$$
\begin{aligned}
d_{\alpha}(u, v)= & d_{\alpha}(T u, T v) \\
\leq & \alpha d_{\alpha}(u, v)+\beta d_{\alpha}(u, T v)+\gamma d_{\alpha}(v, T u)+\delta d_{\alpha}(v, T v) \\
& +\eta \frac{d_{\alpha}(v, T v)\left[1+d_{\alpha}(u, T u)\right]}{1+d_{\alpha}(u, v)}+\lambda \frac{d_{\alpha}(v, T v)+d_{\alpha}(v, T u)}{1+d_{\alpha}(v, T v) d_{\alpha}(v, T u)} \\
& +\mu \frac{d_{\alpha}(u, T u)\left[1+d_{\alpha}(v, T u)\right]}{1+d_{\alpha}(u, v)+d_{\alpha}(v, T v)} \\
= & (\alpha+\beta+\gamma) d_{\alpha}(u, v)+\lambda d_{\alpha}(v, u),
\end{aligned}
$$

which implies $d_{\alpha}(u, v) \leq(\alpha+\beta+\gamma+\lambda) d_{\alpha}(u, v)$, and this implies $d_{\alpha}(u, v)(1-(\alpha+\beta+\gamma+\lambda)) \leq$ 0 .

Thus $d_{\alpha}(u, v)=0$. Hence $u=v$. This completes the proof. 
If we put $s=1$ in the above theorem, we obtain the following corollary in the generating space of a dislocated metric family.

Corollary 2.3 Let $\left(X, d_{\alpha}\right)$ be a complete $G_{d}$-family, $A$ and $B$ be non-empty closed subsets of $X$. Let $T: A \cup B \rightarrow A \cup B$ be a continuous cyclic mapping satisfying the following condition:

$$
\begin{aligned}
d_{\alpha}(T x, T y) \leq & \alpha d_{\alpha}(x, y)+\beta d_{\alpha}(x, T y)+\gamma d_{\alpha}(y, T x)+\delta d_{\alpha}(y, T y) \\
& +\eta \frac{d_{\alpha}(y, T y)\left[1+d_{\alpha}(x, T x)\right]}{1+d_{\alpha}(x, y)}+\lambda \frac{d_{\alpha}(y, T y)+d_{\alpha}(y, T x)}{1+d_{\alpha}(y, T y) d_{\alpha}(y, T x)} \\
& +\mu \frac{d_{\alpha}(x, T x)\left[1+d_{\alpha}(y, T x)\right]}{1+d_{\alpha}(x, y)+d_{\alpha}(y, T y)},
\end{aligned}
$$

where $\alpha, \beta, \gamma, \delta, \eta, \lambda, \mu \geq 0$ with $\alpha+2 \beta+2 \gamma+\delta+\eta+\lambda+\mu<1$ and for all $x, y \in X$. Then $T$ has a unique fixed point in $A \cap B$.

If we put $d$ instead of $d_{\alpha}$ in the above theorem, we obtain the following corollary in the $b$-dislocated metric space.

Corollary 2.4 Let $\left(X, b_{d}\right)$ be a complete $b$-dislocated metric space, $A$ and $B$ be non-empty closed subsets of $X$. Let $T: A \cup B \rightarrow A \cup B$ be a continuous cyclic mapping satisfying the following condition:

$$
\begin{aligned}
b_{d}(T x, T y) \leq & \alpha b_{d}(x, y)+\beta b_{d}(x, T y)+\gamma b_{d}(y, T x)+\delta b_{d}(y, T y) \\
& +\eta \frac{b_{d}(y, T y)\left[1+b_{d}(x, T x)\right]}{1+b_{d}(x, y)}+\lambda \frac{b_{d}(y, T y)+b_{d}(y, T x)}{1+b_{d}(y, T y) b_{d}(y, T x)} \\
& +\mu \frac{b_{d}(x, T x)\left[1+b_{d}(y, T x)\right]}{1+b_{d}(x, y)+b_{d}(y, T y)},
\end{aligned}
$$

where $\alpha, \beta, \gamma, \delta, \eta, \lambda, \mu \geq 0$ with $s \alpha+\left(s^{2}+s\right) \beta+2 s^{2} \gamma+\delta+\eta+\lambda+s \mu<1$ and for all $x, y \in X$. Then $T$ has a unique fixed point in $A \cap B$.

Example 2.5 Let $X=[0,1]$ and $T: A \cup B \rightarrow A \cup B$ defined by $T x=\frac{x}{3}$. Suppose that $A=$ $B=[0,1]$. Define the function $d: X \times X \rightarrow \mathbb{R}$ by $d(x, y)=|x-y|^{2}+\frac{x}{5}+\frac{y}{5}$. Clearly $d$ is a $b$-dislocated metric on $X$ and also $T$ is cyclic mapping on $X$ since $T(A) \subset B$ and $T(B) \subset A$.

Now, consider

$$
\begin{aligned}
d(T x, T y) & =|T x-T y|^{2}+\frac{T x}{5}+\frac{T y}{5} \\
& =\left|\frac{x}{3}-\frac{y}{3}\right|^{2}+\frac{x}{15}+\frac{y}{15} \\
& =\frac{1}{9}|x-y|^{2}+\frac{1}{15} x+\frac{1}{15} y \\
& \leq \frac{1}{3}\left[|x-y|^{2}+\frac{x}{5}+\frac{y}{5}\right] \\
& \leq \alpha d(x, y)
\end{aligned}
$$

for $\frac{1}{3} \leq \alpha<\frac{1}{s}<1$ and $\beta=\gamma=\delta=\eta=\lambda=\mu=0$. 
Thus $T$ satisfies all the conditions of above corollary and ' 0 ' is the unique fixed point.

If we put $d$ instead of $d_{\alpha}$ and $s=1$ in the above theorem, we obtain the following corollary in a complete dislocated metric space.

Corollary 2.6 Let $(X, d)$ be a complete dislocated metric space, $A$ and $B$ be non-empty closed subsets of $X$. Let $T: A \cup B \rightarrow A \cup B$ be a continuous cyclic mapping satisfying the following condition:

$$
\begin{aligned}
d(T x, T y) \leq & \alpha d(x, y)+\beta d(x, T y)+\gamma d(y, T x)+\delta d(y, T y) \\
& +\eta \frac{d(y, T y)[1+d(x, T x)]}{1+d(x, y)}+\lambda \frac{d(y, T y)+d(y, T x)}{1+d(y, T y) d(y, T x)} \\
& +\mu \frac{d(x, T x)[1+d(y, T x)]}{1+d(x, y)+d(y, T y)},
\end{aligned}
$$

where $\alpha, \beta, \gamma, \delta, \eta, \lambda, \mu \geq 0$ with $\alpha+2 \beta+2 \gamma+\delta+\eta+\lambda+\mu<1$ and for all $x, y \in X$. Then $T$ has a unique fixed point in $A \cap B$.

We can find more interesting results in the view of dislocated metric space (see for example [37-40]).

Example 2.7 Let $X=[-1,1]$ and $T: A \cup B \rightarrow A \cup B$ defined by $T x=\frac{-x}{15}$. Suppose that $A=[-1,0]$ and $B=[0,1]$. Define the function $d: X \times X \rightarrow \mathbb{R}$ by $d(x, y)=|x-y|+|x|+|y|$. Hence $d$ is a complete dislocated metric on $X$.

Now consider

$$
\begin{aligned}
d(T x, T y) & =d\left(\frac{-x}{15}, \frac{-y}{15}\right) \\
& =\frac{1}{15}|y-x|+\frac{|x|}{15}+\frac{|y|}{15} \\
& =\frac{1}{15}[|x-y|+|x|+|y|] \\
& \leq \alpha d(x, y),
\end{aligned}
$$

where $\frac{1}{15} \leq \alpha \leq 1$ and $\beta=\gamma=\delta=\eta=\lambda=\mu=0$.

Thus $T$ satisfy all the conditions of the above corollary and ' 0 ' is a unique fixed point.

Definition 2.8 Let $A$ and $B$ be non-empty subsets of a $G_{b d}$-family $\left(X, d_{\alpha}\right)$. A cyclic map $T: A \cup B \rightarrow A \cup B$ is said to be a Hardy-Rogers cyclic orbital contraction if for some $x \in A$ there exists a $\vartheta \in\left(0, \frac{1}{1+3 s+3 s^{2}}\right)$ such that

$$
\begin{aligned}
d_{\alpha}\left(T^{2 n} x, T y\right) \leq & \vartheta\left[d_{\alpha}\left(T^{2 n-1} x, T^{2 n} x\right)+d_{\alpha}(y, T y)\right. \\
& \left.+d_{\alpha}\left(T^{2 n-1} x, T y\right)+d_{\alpha}\left(y, T^{2 n} x\right)+d_{\alpha}\left(T^{2 n-1} x, y\right)\right]
\end{aligned}
$$

Theorem 2.9 Let $A$ and $B$ be non-empty closed subsets of a complete $G_{b d}$-family $\left(X, d_{\alpha}\right)$ and $T: A \cup B \rightarrow A \cup B$ be a Hardy-Rogers cyclic orbital contraction. Then $T$ has a unique fixed point $z$ in $A \cap B$. 
Proof Take $x \in A$. Since $T: A \cup B \rightarrow A \cup B$ is a Hardy-Rogers cyclic orbital contraction,

$$
\begin{aligned}
d_{\alpha}\left(T^{2} x, T x\right) \leq & \vartheta\left[d_{\alpha}\left(T x, T^{2} x\right)+d_{\alpha}(x, T x)+d_{\alpha}(T x, T x)+d_{\alpha}\left(x, T^{2} x\right)+d_{\alpha}(T x, x)\right] \\
\leq & \vartheta\left[d_{\alpha}\left(T x, T^{2} x\right)+d_{\alpha}(x, T x)+s\left(d_{\beta}(T x, x)+d_{\beta}(x, T x)\right)\right. \\
& \left.+s\left(d_{\beta}(x, T x)+d_{\beta}\left(T x, T^{2} x\right)\right)+d_{\alpha}(T x, x)\right] \\
= & {[\vartheta+(2 s+s) \vartheta+\vartheta] d_{\alpha}(x, T x)+(\vartheta+s \vartheta) d_{\alpha}\left(T x, T^{2} x\right), }
\end{aligned}
$$

which implies

$$
\begin{aligned}
& d_{\alpha}(\left.T^{2} x, T x\right) \leq \frac{\vartheta(2+3 s)}{1-\vartheta(1+s)} d_{\alpha}(x, T x), \\
& d_{\alpha}\left(T^{3} x, T^{2} x\right) \\
& \leq \vartheta \\
& \quad+d_{\alpha}\left(T^{2} x, T^{3} x\right)+d_{\alpha}\left(T x, T^{2} x\right)+d_{\alpha}\left(T^{2} x, T^{2} x\right) \\
&\left.\quad+d_{\alpha}\left(T x, T^{3} x\right)+d_{\alpha}\left(T^{2} x, T x\right)\right] \\
&=\vartheta d_{\alpha}\left(T^{2} x, T^{3} x\right)+\vartheta d_{\alpha}\left(T^{2} x, T x\right)+\vartheta\left[s\left(d_{\beta}\left(T^{2} x, T x\right)+d_{\beta}\left(T x, T^{2} x\right)\right)\right] \\
& \quad+\vartheta s\left[\left(d_{\beta}\left(T x, T^{2} x\right)+d_{\beta}\left(T^{2} x, T^{3} x\right)\right]+\vartheta d_{\alpha}\left(T^{2} x, T x\right)\right. \\
&=(\vartheta+2 s \vartheta+\vartheta s+\vartheta) d_{\alpha}\left(T^{2} x, T x\right)+(\vartheta+s \vartheta) d_{\alpha}\left(T^{3} x, T^{2} x\right) .
\end{aligned}
$$

Thus

$$
\begin{gathered}
(1-\vartheta-\vartheta s) d_{\alpha}\left(T^{3} x, T^{2} x\right) \leq(2 \vartheta+3 s \vartheta) d_{\alpha}\left(T^{2} x, T x\right) \\
\Rightarrow \quad d_{\alpha}\left(T^{3} x, T^{2} x\right) \leq \frac{\vartheta(2+3 s)}{1-\vartheta(1+s)} d_{\alpha}\left(T^{2} x, T x\right)
\end{gathered}
$$

which yields

$$
\begin{aligned}
d_{\alpha}\left(T^{3} x, T^{2} x\right) & \leq k d_{\alpha}\left(T^{2} x, T x\right) \\
& \leq k^{2} d_{\alpha}(T x, x) ; \quad \text { where } k=\frac{\vartheta(2+3 s)}{1-\vartheta(1+s)}
\end{aligned}
$$

By repeating the same process, we get

$$
d_{\alpha}\left(T^{n+1} x, T^{n} x\right) \leq k^{n} d_{\alpha}(T x, x)
$$

Taking the limits as $n \rightarrow \infty$, we get

$$
\lim _{n \rightarrow \infty} d_{\alpha}\left(T^{n+1} x, T^{n} x\right)=0, \quad \text { since } 0<k<1 .
$$

We now claim that $\lim _{n \rightarrow \infty} d_{\alpha}\left(T^{n} x, T^{m} x\right)=0$ for $m>n$.

For $m, n \in \mathbb{N}$ with $m>n$, we have

$$
\begin{aligned}
d_{\alpha}\left(T^{n} x, T^{m} x\right) & \leq s\left[d_{\beta}\left(T^{n} x, T^{n+1} x\right)+d_{\beta}\left(T^{n+1} x, T^{m} x\right)\right] \\
& \leq s d_{\beta}\left(T^{n} x, T^{n+1} x\right)+s^{2} d_{\beta}\left(T^{n+1} x, T^{n+2} x\right)+s^{2} d_{\beta}\left(T^{n+2} x, T^{m} x\right)
\end{aligned}
$$




$$
\begin{aligned}
\leq & s d_{\beta}\left(T^{n} x, T^{n+1} x\right)+s^{2} d_{\beta}\left(T^{n+1} x, T^{n+2} x\right)+s^{3} d_{\beta}\left(T^{n+2} x, T^{n+3} x\right) \\
& +s^{4} d_{\beta}\left(T^{n+3} x, T^{n+4} x\right)+\cdots \\
& \vdots \\
\leq & s k^{n} d_{\alpha}(T x, x)+s^{2} k^{n+1} d_{\alpha}(T x, x)+s^{3} k^{n+2} d_{\alpha}(T x, x)+\cdots \\
\leq & s k^{n}\left(1+s k+(s k)^{2}+\cdots\right) d_{\alpha}(T x, x) \\
\leq & \frac{s k^{n}}{1-s k} d_{\alpha}(T x, x) \\
\leq & \frac{(s k)^{n}}{1-s k} d_{\alpha}(T x, x) .
\end{aligned}
$$

Since $s k<1$ and letting $n \rightarrow \infty$, we get $\lim _{n \rightarrow \infty} d_{\alpha}\left(T^{n} x, T^{m} x\right)=0$.

Thus $\left\{T^{n} x\right\}$ is a Cauchy sequence.

Since $\left(X, d_{\alpha}\right)$ is a complete generating $b$-dislocated metric family, there exists $z \in A \cup B$ such that $\lim _{n \rightarrow \infty} T^{n} x=z$. Now $\left\{T^{2 n} x\right\}$ is a sequence in $A$ and $\left\{T^{2 n-1} x\right\}$ is a sequence in $B$, and also both converge to $z$. Since $A$ and $B$ are closed, $z \in A \cap B$, and so $A \cap B$ in non-empty.

Now consider

$$
\begin{aligned}
d_{\alpha}(z, T z)= & \lim _{n \rightarrow \infty} d_{\alpha}\left(T^{n} x, T z\right) \\
\leq & \vartheta \lim _{n \rightarrow \infty}\left[d_{\alpha}\left(T^{n-1} x, T^{n} x\right)+d_{\alpha}(z, T z)+d_{\alpha}\left(T^{n-1} x, T z\right)\right. \\
& \left.+d_{\alpha}\left(z, T^{n} x\right)+d_{\alpha}\left(T^{n-1} x, z\right)\right] \\
\leq & \vartheta d_{\alpha}(z, T z)
\end{aligned}
$$

which implies $(1-\vartheta) d_{\alpha}(z, T z) \leq 0$.

Since $0<\vartheta<1, d_{\alpha}(z, T z)=0$.

Hence $z=T z$. Thus $z$ is a fixed point of $T$.

Finally, to prove $z$ is a unique fixed point of $T$, let us assume, on the contrary, that $z^{*}$ is another fixed point of $T$, i.e. $T z^{*}=z^{*}$.

Note that, if $z^{*}$ is a fixed point of $T$, then $d_{\alpha}\left(z^{*}, z^{*}\right)=0$. We have

$$
\begin{aligned}
d_{\alpha}\left(z^{*}, z^{*}\right) & =d_{\alpha}\left(T z^{*}, T z^{*}\right) \\
& \leq \vartheta\left[d_{\alpha}\left(z^{*}, T z^{*}\right)+d_{\alpha}\left(z^{*}, T z^{*}\right)+d_{\alpha}\left(z^{*}, T z^{*}\right)+d_{\alpha}\left(z^{*}, T z^{*}\right)+d_{\alpha}\left(z^{*}, z^{*}\right)\right] \\
& \leq 5 \vartheta d_{\alpha}\left(z^{*}, z^{*}\right) .
\end{aligned}
$$

This implies $(1-5 \vartheta) d_{\alpha}\left(z^{*}, z^{*}\right) \leq 0$.

Since $1-5 \vartheta>0, d_{\alpha}\left(z^{*}, z^{*}\right)=0$.

By the cyclic property of $T$, we have $z, z^{*} \in A \cap B$. From the Hardy-Rogers cyclic orbital contraction, we have

$$
\begin{aligned}
d_{\alpha}\left(z, z^{*}\right) & =d_{\alpha}\left(z, T z^{*}\right) \\
& =\lim _{n \rightarrow \infty} d_{\alpha}\left(T^{2 n} x, T z^{*}\right) \\
& \leq \vartheta \lim _{n \rightarrow \infty}\left[d_{\alpha}\left(T^{2 n-1} x, T^{2 n} x\right)+d_{\alpha}\left(z^{*}, T z^{*}\right)+d_{\alpha}\left(T^{2 n-1} x, T z^{*}\right)\right.
\end{aligned}
$$




$$
\begin{aligned}
& \left.+d_{\alpha}\left(z^{*}, T^{2 n} x\right)+d_{\alpha}\left(T^{2 n-1} x, z^{*}\right)\right] \\
\leq & \vartheta d_{\alpha}\left(z, z^{*}\right),
\end{aligned}
$$

which implies $(1-\vartheta) d_{\alpha}\left(z, z^{*}\right)=0$. Hence $z=z^{*}$.

Thus $z$ is a unique fixed point of $T$. This completes the proof of the theorem.

If we put $s=1$ in the above Theorem 2.9, we obtain the following corollary in the generating space of a dislocated metric family.

Corollary 2.10 Let $A$ and $B$ be non-empty closed subsets of a complete $G_{d}$-family $\left(X, d_{\alpha}\right)$ and $T: A \cup B \rightarrow A \cup B$ be a Hardy-Rogers cyclic orbital contraction. Then $T$ has a unique fixed point $z \in A \cap B$.

If we put $d$ instead of $d_{\alpha}$ in the above theorem, we obtain the following corollary in a $b$-dislocated metric space.

Corollary 2.11 Let $A$ and $B$ be non-empty closed subsets of a complete b-dislocated metric space $\left(X, b_{d}\right)$ and $T: A \cup B \rightarrow A \cup B$ be a Hardy-Rogers cyclic orbital contraction. Then $T$ has a unique fixed point $z \in A \cap B$.

If we put $d$ instead of $d_{\alpha}$ and $s=1$ in the above theorem, we obtain the following corollary in a complete dislocated metric space.

Corollary 2.12 Let $A$ and $B$ be non-empty closed subsets of a complete dislocated metric space $(X, d)$ and $T: A \cup B \rightarrow A \cup B$ be a Hardy-Rogers cyclic orbital contraction. Then $T$ has a unique fixed point $z \in A \cap B$.

Example 2.13 Let $X=\{0,1,2\}$ and $b_{d}: X \times X \rightarrow \mathbb{R}^{+}$by $b_{d}(0,0)=0, b_{d}(1,1)=0, b_{d}(2,2)=$ $1, b_{d}(0,1)=b_{d}(1,0)=0, b_{d}(0,2)=b_{d}(2,0)=1, b_{d}(1,2)=b_{d}(2,1)=2$, which is a $b$-dislocated metric with $s=2$, but not a dislocated metric as triangle inequality does not hold, since $b_{d}(1,2) \not b_{d}(1,0)+b_{d}(0,2)$.

Put $A=\{0,1\}$ and $B=\{1,2\}$. It is clear that $A$ and $B$ are closed subsets of $\left(X, b_{d}\right)$.

Let $X=A \cup B$. Now define $T: A \cup B \rightarrow A \cup B$ by $T 0=1, T 1=1$, and $T 2=0$. Then $T(A)=B$ and $T(B)=A$, so $T$ is a cyclic map on $A \cup B$.

Fix $x=0 \in A$ and for every $y \in A$, we have $T^{2 n} x=1, T^{2 n-1} x=1$.

Thus the Hardy-Rogers cyclic orbital contraction,

$$
\begin{aligned}
b_{d}\left(T^{2 n} x, T y\right) \leq & \vartheta\left[b_{d}\left(T^{2 n-1} x, T^{2 n} x\right)+b_{d}(y, T y)+b_{d}\left(T^{2 n-1} x, T y\right)\right. \\
& \left.+b_{d}\left(y, T^{2 n} x\right)+b_{d}\left(T^{2 n-1} x, y\right)\right]
\end{aligned}
$$

for each $n \in \mathbb{N}$ and for each $y \in A$, satisfies for $\vartheta \in\left(0, \frac{1}{19}\right)$.

Hence from Corollary 2.10, $T$ has the unique fixed point and it is observed that $z=1 \epsilon$ $A \cap B$ is the unique fixed point of $T$. 
Example 2.14 Let $A=B=X=[0,1]$ with the complete dislocated metric $d(x, y)=x+y$. Define $T: X \rightarrow X$ as follows:

$$
T x= \begin{cases}0, & \text { if } x \in\left[0, \frac{1}{3}\right), \\ 0.1, & \text { if } x \in\left[\frac{1}{3}, 1\right] .\end{cases}
$$

Fix any $x \in\left[0, \frac{1}{3}\right), T x=0, T^{2} x=0, T^{3} x=0, \ldots, T^{n} x=0 \forall n$, and for every $y \in[0,1]$ we have

$$
\begin{aligned}
& T y= \begin{cases}0, & \text { if } y \in\left[0, \frac{1}{3}\right), \\
0.1, & \text { if } y \in\left[\frac{1}{3}, 1\right]\end{cases} \\
& d\left(T^{2 n} x, T y\right)= \begin{cases}d(0,0)=0, & \text { if } y \in\left[0, \frac{1}{3}\right), \\
d(0,0.1)=0.1, & \text { if } y \in\left[\frac{1}{3}, 1\right]\end{cases} \\
& d\left(T^{2 n} x, y\right)=d(0, y)=y \quad \text { and } \quad d(T y, y)= \begin{cases}d(0, y)=y, & \text { if } y \in\left[0, \frac{1}{3}\right), \\
d(0.1, y)=0.1+y, & \text { if } y \in\left[\frac{1}{3}, 1\right],\end{cases} \\
& d\left(T^{2 n-1} x, T^{2 n} x\right)=d(0,0)=0, \quad \text { and } \\
& d\left(T^{2 n-1} x, T y\right)= \begin{cases}d(0,0)=0, & \text { if } y \in\left[0, \frac{1}{3}\right), \\
d(0,0.1)=0.1, & \text { if } y \in\left[\frac{1}{3}, 1\right]\end{cases} \\
& d\left(y, T^{2 n} x\right)=d(y, 0)=y .
\end{aligned}
$$

Thus, for each $n \in \mathbb{N}$ and for each $y \in[0,1]$ the Hardy-Rogers cyclic orbital contraction,

$$
d\left(T^{2 n} x, T y\right) \leq \vartheta\left[d\left(T^{2 n-1} x, T^{2 n} x\right)+d(y, T y)+d\left(T^{2 n-1} x, T y\right)+d\left(y, T^{2 n} x\right)+d\left(T^{2 n-1} x, y\right)\right],
$$

is satisfied for $0.03 \leq \vartheta<0.1$. Thus by Corollary 2.12, $T$ has the unique fixed point in $A \cap B$. In fact ' 0 ' is the unique fixed point for $T$.

Definition 2.15 Let $\left(X, d_{\alpha}\right)$ be a complete $G_{b d}$-family. A self-mapping $T: X \rightarrow X$ is said to be a Hardy-Roger F-contraction if there exists $\tau>0$ such that

$$
\begin{aligned}
\frac{1}{2} d_{\alpha}(x, T x) & <d_{\alpha}(x, y) \\
\Rightarrow \quad \tau & +F\left(d_{\alpha}(T x, T y)\right) \leq \alpha F\left(d_{\alpha}(x, y)\right)+\beta F\left(d_{\alpha}(x, T x)\right)+\gamma F\left(d_{\alpha}(y, T y)\right) \\
& +\delta F\left(d_{\alpha}(x, T y)\right)+\eta F\left(d_{\alpha}(y, T x)\right)
\end{aligned}
$$

for all $x, y \in X$ with $x \neq y$, where $\gamma \neq 1, \eta \geq 0$ such that $\alpha+\beta+\gamma+2 s \delta+2 s \eta=1$ and $F: \mathbb{R}^{+} \rightarrow \mathbb{R}$ is a mapping satisfying the following conditions:

( $\left.\mathrm{F}^{\prime}\right) \quad F$ is an order embedding, i.e. for all $\alpha, \beta \in \mathbb{R}^{+}$we have

$$
\alpha \leq \beta, \quad F(\alpha) \leq F(\beta),
$$

and also sub-additive. 
$\left(\mathrm{F}^{\prime \prime}\right)$ For any sequence $\left\{\alpha_{n}\right\}_{n=1}^{\infty}$ of positive real numbers,

$$
\lim _{n \rightarrow \infty} \alpha_{n}=0 \quad \text { if and only if } \quad \lim _{n \rightarrow \infty} F\left(\alpha_{n}\right)=-\infty
$$

Theorem 2.16 Let $\left(X, d_{\alpha}\right)$ be a complete $G_{b d}$-family and $T$ be a Hardy-Roger $F$-contraction. Then $T$ has a fixed point in $X$.

Proof For an arbitrary $x \in X$, we establish a sequence $\left\{x_{n}\right\}$ in the following manner:

$$
x=x_{0} \quad \text { and } \quad x_{n+1}=T x_{n} \quad \forall n \in \mathbb{N} .
$$

If there exists $n_{0} \in \mathbb{N}$ such that $d_{\alpha}\left(x_{n_{0}}, x_{n_{0}+1}\right)=0$, then $v=x_{n_{0}}$ is the needed fixed point of $T$, which completes the proof. Accordingly, we assume that $0<d_{\alpha}\left(x_{n}, x_{n+1}\right)$ for every $n \in \mathbb{N}$. Hence we have

$$
\frac{1}{2} d_{\alpha}\left(x_{n}, T x_{n}\right)=\frac{1}{2} d_{\alpha}\left(x_{n}, x_{n+1}\right)<d_{\alpha}\left(x_{n}, x_{n+1}\right), \quad n \in \mathbb{N} .
$$

From the hypothesis of the theorem, we have

$$
\begin{aligned}
\tau+F\left(d_{\alpha}\left(T x_{n}, T x_{n+1}\right)\right) \leq & \alpha F d_{\alpha}\left(x_{n}, x_{n+1}\right)+\beta F d_{\alpha}\left(x_{n}, x_{n+1}\right)+\gamma F d_{\alpha}\left(x_{n+1}, x_{n+2}\right) \\
& +\delta F d_{\alpha}\left(x_{n}, x_{n+2}\right)+\eta F d_{\alpha}\left(x_{n+1}, x_{n+1}\right) \\
\tau+F\left(d_{\alpha}\left(x_{n+1}, x_{n+2}\right)\right) \leq & \alpha F d_{\alpha}\left(x_{n}, x_{n+1}\right)+\beta F d_{\alpha}\left(x_{n}, x_{n+1}\right)+\gamma F d_{\alpha}\left(x_{n+1}, x_{n+2}\right) \\
& +s \delta F d_{\beta}\left(x_{n}, x_{n+1}\right)+s \delta F d_{\beta}\left(x_{n+1}, x_{n+2}\right)+s \eta F d_{\beta}\left(x_{n+1}, x_{n+2}\right) \\
& +s \eta F d_{\beta}\left(x_{n+2}, x_{n+1}\right) \\
\tau+(1-\gamma-s \delta-2 s \eta) F( & \left.d_{\alpha}\left(x_{n+1}, x_{n+2}\right)\right) \leq(\alpha+\beta+s \delta) d_{\alpha}\left(x_{n}, x_{n+1}\right) .
\end{aligned}
$$

Since $\alpha+\beta+\gamma+2 s \delta+2 s \eta=1$, we obtain

$$
\begin{aligned}
F\left(d_{\alpha}\left(x_{n+1}, x_{n+2}\right)\right) & \leq \frac{\alpha+\beta+s \delta}{1-\gamma-s \delta-2 s \eta} F d_{\alpha}\left(x_{n}, x_{n+1}\right)-\frac{\tau}{1-\gamma-s \delta-2 s \eta} \\
& <F d_{\alpha}\left(x_{n}, x_{n+1}\right) .
\end{aligned}
$$

Thus, $\left\{d_{\alpha}\left(x_{n}, x_{n+1}\right)\right\}_{n=1}^{\infty}$ is a non-increasing sequence of real numbers which is bounded below. This implies that $\left\{d_{\alpha}\left(x_{n}, x_{n+1}\right)\right\}_{n=1}^{\infty}$ converges and

$$
\lim _{n \rightarrow \infty} d_{\alpha}\left(x_{n}, x_{n+1}\right)=\beta=\inf \left\{d_{\alpha}\left(x_{n}, x_{n+1}\right): n \in \mathbb{N}\right\}
$$

We shall show that $\beta=0$. Suppose, on the contrary, that $\beta>0$. For every $\epsilon>0$ there exists $m \in \mathbb{N}$, such that

$$
d_{\alpha}\left(x_{m}, T x_{m}\right)<\beta+\epsilon .
$$

Hence from $\left(\mathrm{F}^{\prime}\right)$, we get

$$
F\left(d_{\alpha}\left(x_{m}, T x_{m}\right)\right)<F(\beta+\epsilon) .
$$


On the other side, we have

$$
\frac{1}{2 s} d_{\alpha}\left(x_{m}, T x_{m}\right)<d_{\alpha}\left(x_{m}, T x_{m}\right) .
$$

Due to the supposition of the theorem, we get

$$
\begin{aligned}
\tau+F\left(d_{\alpha}\left(T x_{m}, T^{2} x_{m}\right)\right) \leq & \alpha F d_{\alpha}\left(x_{m}, T x_{m}\right)+\beta F d_{\alpha}\left(x_{m}, T x_{m}\right)+\gamma F d_{\alpha}\left(T x_{m}, T^{2} x_{m}\right) \\
& +\delta F d_{\alpha}\left(x_{m}, T^{2} x_{m}\right)++\eta F d_{\alpha}\left(T x_{m}, T x_{m}\right)
\end{aligned}
$$

which is equivalent to

$$
\tau+(1-\gamma-s \delta-2 s \eta) F\left(d_{\alpha}\left(T x_{m}, T^{2} x_{m}\right)\right) \leq(\alpha+\beta+s \delta) F d_{\alpha}\left(x_{m}, T x_{m}\right),
$$

which implies

$$
\begin{aligned}
F\left(d_{\alpha}\left(T x_{m}, T^{2} x_{m}\right)\right) & \leq F\left(d_{\alpha}\left(x_{m}, T x_{m}\right)\right)-\frac{\tau}{1-\gamma-s \delta-2 s \eta} \\
& <F\left(d_{\alpha}\left(x_{m}, T x_{m}\right)\right) .
\end{aligned}
$$

By continuing the same process, we get

$$
\begin{aligned}
F\left(d_{\alpha}\left(T^{n} x_{m}, T^{n+1} x_{m}\right)\right) & \leq F\left(d_{\alpha}\left(T^{n} x_{m}, T^{n-1} x_{m}\right)\right)-\frac{\tau}{1-\gamma-s \delta-2 s \eta} \\
& \leq F\left(d_{\alpha}\left(T^{n-1} x_{m}, T^{n-2} x_{m}\right)\right)-\frac{2 \tau}{1-\gamma-s \delta-2 s \eta} \\
& \vdots \\
& \leq F\left(d_{\alpha}\left(T x_{m}, x_{m}\right)\right)-\frac{n \tau}{1-\gamma-s \delta-2 s \eta} \\
& <F(\beta+\epsilon)-\frac{n \tau}{1-\gamma-s \delta-2 s \eta},
\end{aligned}
$$

which yields $\lim _{n \rightarrow \infty} F\left(d_{\alpha}\left(T^{n} x_{m}, T^{n+1} x_{m}\right)\right)=-\infty$.

From $\left(\mathrm{F}^{\prime \prime}\right)$, we have $\lim _{n \rightarrow \infty} d_{\alpha}\left(T^{n} x_{m}, T^{n+1} x_{m}\right)=0$ and thus, there exists $N_{1} \in \mathbb{N}$ such that $d_{\alpha}\left(T^{n} x_{m}, T^{n+1} x_{m}\right)<\beta ; \forall n \geq N_{1}$.

This yields

$$
d_{\alpha}\left(x_{m+n}, T x_{m+n}\right)<\beta, \quad \forall n \geq N_{1} .
$$

This contradicts the definition of $\beta$. Thus $\beta=0$.

Therefore $\lim _{n \rightarrow \infty} d_{\alpha}\left(x_{n}, x_{n+1}\right)=0$.

Next, our aim is to prove $\lim _{n, m \rightarrow \infty} d_{\alpha}\left(x_{n}, x_{m}\right)=0$.

Suppose, on the contrary, that there exist $\epsilon>0$ and sequences $\left\{\psi_{n}\right\}_{n=1}^{\infty}$ and $\left\{\varphi_{n}\right\}_{n=1}^{\infty}$ of natural numbers such that $\psi(n)>\varphi(n)>n, d_{\alpha}\left(x_{\psi(n)}, x_{\varphi(n)}\right) \geq \epsilon$,

$$
d_{\alpha}\left(x_{\psi(n)-1}, x_{\varphi(n)}\right)<\frac{\epsilon}{s}, \quad \forall n \in \mathbb{N} .
$$


By the definition of a generating space of a $b$-dislocated metric family,

$$
\begin{aligned}
\epsilon & \leq d_{\alpha}\left(x_{\psi(n)}, x_{\varphi(n)}\right) \\
& \leq s\left[d_{\beta}\left(x_{\psi(n)}, x_{\psi(n)-1}\right)+d_{\beta}\left(x_{\psi(n)-1}, x_{\varphi(n)}\right)\right] \\
& \leq s d_{\alpha}\left(x_{\psi(n)}, x_{\varphi(n)-1}\right)+\epsilon, \quad \forall n \in \mathbb{N} .
\end{aligned}
$$

From (30) and (32) and the squeezing theorem,

$$
\lim _{n \rightarrow \infty} d_{\alpha}\left(x_{\psi(n)}, x_{\varphi(n)}\right)=\epsilon
$$

From (30), (31), and (33) there exists $N_{2} \in \mathbb{N}$ such that

$$
\frac{1}{2} d_{\alpha}\left(x_{\psi(n)}, T x_{\psi(n)}\right)<\frac{1}{2} \epsilon<\epsilon \leq d_{\alpha}\left(x_{\psi(n)}, x_{\varphi(n)}\right)<2 \epsilon, \quad \forall n \in N_{2} .
$$

Hence from (34) and $\left(\mathrm{F}^{\prime}\right)$, and the hypothesis of the theorem, we have

$$
\begin{aligned}
\tau+ & F\left(d_{\alpha}\left(T x_{\psi(n)}, T x_{\varphi(n)}\right)\right) \\
\leq & \alpha F\left(d_{\alpha}\left(x_{\psi(n)}, x_{\varphi(n)}\right)\right)+\beta F\left(d_{\alpha}\left(x_{\psi(n)}, T x_{\psi(n)}\right)\right)+\gamma F\left(d_{\alpha}\left(x_{\varphi(n)}, T x_{\varphi(n)}\right)\right) \\
& +\delta F\left(d_{\alpha}\left(x_{\psi(n)}, T x_{\varphi(n)}\right)\right)+\eta F\left(d_{\alpha}\left(x_{\varphi(n)}, T x_{\psi(n)}\right)\right) \\
\leq & \alpha F(2 \epsilon)+\beta F\left(d_{\alpha}\left(x_{\psi(n)}, T x_{\psi(n)}\right)\right)+\gamma F\left(d_{\alpha}\left(x_{\varphi(n)}, T x_{\varphi(n)}\right)\right)+\delta F(2 \epsilon) \\
& +\eta s F\left(d_{\alpha}\left(x_{\varphi(n)}, x_{\varphi(n)+1}\right)\right)+\eta s F\left(d_{\alpha}\left(x_{\varphi(n)}, T x_{\psi(n)}\right)\right), \\
\tau+ & (1-\eta s) F\left(d_{\alpha}\left(T x_{\psi(n)}, T x_{\varphi(n)}\right)\right) \\
\leq & \alpha F(2 \epsilon)+\beta F\left(d_{\alpha}\left(x_{\psi(n)}, T x_{\psi(n)}\right)\right)+\gamma F\left(d_{\alpha}\left(x_{\varphi(n)}, T x_{\varphi(n)}\right)\right)+\delta F(2 \epsilon) \\
& +\eta s F\left(d_{\alpha}\left(x_{\varphi(n)}, x_{\varphi(n)+1}\right)\right) .
\end{aligned}
$$

This implies

$$
\lim _{n \rightarrow \infty} F\left(d_{\alpha}\left(T x_{\psi(n)}, T x_{\varphi(n)}\right)\right)=-\infty
$$

Thus,

$$
\lim _{n \rightarrow \infty} d_{\alpha}\left(T x_{\psi(n)}, T x_{\varphi(n)}\right)=0 \quad \text { iff } \quad \lim _{n \rightarrow \infty} d_{\alpha}\left(x_{\psi(n)+1}, x_{\varphi(n)+1}\right)=0 .
$$

Hence $\lim _{n, m \rightarrow \infty} d_{\alpha}\left(x_{n}, x_{m}\right)=0$.

Therefore $\left\{x_{n}\right\}_{n=1}^{\infty}$ is a Cauchy sequence in $X$. From the completeness of $\left(X, d_{\alpha}\right)$ there exists $z \in X$ such that

$$
d_{\alpha}(z, z)=\lim _{n \rightarrow \infty} d_{\alpha}\left(x_{n}, z\right)=\lim _{n, m \rightarrow \infty} d_{\alpha}\left(x_{n}, x_{m}\right)=0
$$

Subsequently, we shall prove that, for every $n \in \mathbb{N}$,

$$
\frac{1}{2 s} d_{\alpha}\left(x_{n}, T x_{n}\right)<d_{\alpha}\left(x_{n}, z\right) \quad \text { or } \quad \frac{1}{2 s} d_{\alpha}\left(T x_{n}, T^{2} x_{n}\right)<d_{\alpha}\left(T x_{n}, z\right), \quad \forall n \in \mathbb{N} .
$$


Arguing by contradiction, we suppose that there exists $m \in \mathbb{N}$ such that

$$
\frac{1}{2 s} d_{\alpha}\left(x_{m}, T x_{m}\right) \geq d_{\alpha}\left(x_{m}, z\right) \text { and } \frac{1}{2 s} d_{\alpha}\left(T x_{m}, T^{2} x_{m}\right) \geq d_{\alpha}\left(T x_{m}, z\right)
$$

Thus

$$
d_{\alpha}\left(T x_{m}, T^{2} x_{m}\right)<d_{\alpha}\left(x_{m}, T x_{m}\right) .
$$

Now, take

$$
\begin{aligned}
d_{\alpha}\left(x_{m}, T x_{m}\right) & \leq s\left[d_{\beta}\left(x_{m}, v\right)+d_{\beta}\left(v, T x_{m}\right)\right] \\
& \leq \frac{1}{2} d_{\alpha}\left(x_{m}, T x_{m}\right)+\frac{1}{2} d_{\alpha}\left(T x_{m}, T^{2} x_{m}\right) \\
& <\frac{1}{2} d_{\alpha}\left(x_{m}, T x_{m}\right)+\frac{1}{2} d_{\alpha}\left(x_{m}, T x_{m}\right) \\
& =d_{\alpha}\left(x_{m}, T x_{m}\right) .
\end{aligned}
$$

Evidently, this is a contradiction. Hence, the inequality (37) is satisfied. Concerning the supposition of the theorem, (37) implies that either

$$
\begin{aligned}
\tau+F\left(d_{\alpha}\left(T x_{n}, T z\right)\right) \leq & \alpha F\left(d_{\alpha}\left(x_{n}, z\right)\right)+\beta F\left(d_{\alpha}\left(x_{n}, T x_{n}\right)\right) \\
& +\gamma F\left(d_{\alpha}(z, T z)\right)+\delta F\left(d_{\alpha}\left(x_{n}, T z\right)\right)+\eta F\left(d_{\alpha}\left(z, T x_{n}\right)\right)
\end{aligned}
$$

or

$$
\begin{aligned}
\tau+F\left(d_{\alpha}\left(T^{2} x_{n}, T z\right)\right) \leq & \alpha F\left(d_{\alpha}\left(T x_{n}, z\right)\right)+\beta F\left(d_{\alpha}\left(T x_{n}, T^{2} x_{n}\right)\right) \\
& +\gamma F\left(d_{\alpha}(z, T z)\right)+\delta F\left(d_{\alpha}\left(T x_{n}, T z\right)\right)+\eta F\left(d_{\alpha}\left(z, T^{2} x_{n}\right)\right) .
\end{aligned}
$$

Case (i): By taking the limits as $n \rightarrow \infty$ in (41), we get

$$
\lim _{n \rightarrow \infty} F\left(d_{\alpha}\left(T x_{n}, T z\right)\right)=-\infty
$$

which implies

$$
\lim _{n \rightarrow \infty} d_{\alpha}\left(T x_{n}, T z\right)=0
$$

Now,

$$
d_{\alpha}(z, T z) \leq s\left[d_{\beta}\left(z, T x_{n}\right)+d_{\beta}\left(T x_{n}, T z\right)\right]
$$

which implies

$$
d_{\alpha}(z, T z)=0
$$


Thus

$$
z=T z
$$

Case (ii):

$$
\begin{aligned}
F\left(d_{\alpha}\left(T^{2} x_{n}, T z\right)\right) \leq & \tau+F\left(d_{\alpha}\left(T^{2} x_{n}, T z\right)\right) \\
\leq & \alpha F\left(d_{\alpha}\left(T x_{n}, z\right)\right)+\beta F\left(d_{\alpha}\left(T x_{n}, T^{2} x_{n}\right)\right) \\
& +\gamma F\left(d_{\alpha}(z, T z)\right)+\delta F\left(d_{\alpha}\left(T x_{n}, T z\right)\right)+\eta F\left(d_{\alpha}\left(z, T^{2} x_{n}\right)\right) .
\end{aligned}
$$

Thus

$$
\lim _{n \rightarrow \infty} F\left(d_{\alpha}\left(T^{2} x_{n}, T z\right)\right)=-\infty
$$

From $\left(\mathrm{F}^{\prime \prime}\right)$, we get

$$
\lim _{n \rightarrow \infty} d_{\alpha}\left(T^{2} x_{n}, T z\right)=0
$$

Consider

$$
\begin{aligned}
d_{\alpha}(z, T z) & \leq s d_{\beta}\left(z, T^{2} x_{n}\right)+s d_{\beta}\left(T^{2} x_{n}, T z\right) \\
& =s d_{\alpha}\left(z, x_{n+2}\right)+s d_{\alpha}\left(T^{2} x_{n}, T z\right)
\end{aligned}
$$

Thus letting $n \rightarrow \infty, d_{\alpha}(z, T z)=0 \Rightarrow z=T z$.

Hence $z$ is a fixed point of $T$.

If we put $s=1$ in Theorem 2.16, we obtain the following corollary in the generating space of a dislocated metric family.

Corollary 2.17 Let $\left(X, d_{\alpha}\right)$ be a complete $G_{d}$-family and $T$ be a Hardy-Roger $F$-contraction. Then $T$ has a fixed point in $X$.

If we put $d$ instead of $d_{\alpha}$ in the above Theorem 2.16, we obtain the following corollary in a $b$-dislocated metric space.

Corollary 2.18 Let $\left(X, b_{d}\right)$ be a complete b-dislocated metric space, and $T$ be a HardyRoger $F$-contraction. Then $T$ has a fixed point in $X$.

If we put $d_{\alpha}=d$ and $s=1$ in the above Theorem 2.16, we obtain the following corollary in a complete dislocated metric space.

Corollary 2.19 Let $(X, d)$ be a complete dislocated metric space, and $T$ be a Hardy-Roger $F$-contraction. Then $T$ has a fixed point in $X$. 
Authors' contributions

All authors contributed equally to the writing of this paper. All authors read and approved the final manuscript.

\section{Author details}

'Department of Mathematics, National Institute of Technology, Andhra Pradesh, AP, India. ${ }^{2}$ Department of Mathematics, Nepal Sanskrit University, Valmeeki campus, Exhibition road, Kathmandu, Nepal.

\section{Acknowledgements}

The first author would like to express her sincere gratitude to Mr. Anand Prabhakar for his invaluable support and motivation. The authors would like to express their thanks to the referees for their helpful comments and suggestions.

\section{Received: 14 October 2015 Accepted: 4 January 2016 Published online: 19 January 2016}

\section{References}

1. Banach, S: Sur les operations dans les ensembles abstraits et leur applications aux equations integrales. Fundam. Math. 3, 133-181 (1922)

2. Kannan, R: Some results on fixed points. Bull. Calcutta Math. Soc. 60, 71-76 (1968)

3. Reich, S: Some remarks concerning contraction mappings. Can. Math. Bull. 14, 121-124 (1971)

4. Chatterjea, SK: Fixed-point theorems. C. R. Acad. Bulg. Sci. 25, 727-730 (1972)

5. Ćirić, LB: Generalized contractions and fixed-point theorems. Publ. Inst. Math. (Belgr.) 12(26), $19-26$ (1971)

6. Hardy, GE, Rogers, TD: A generalization of a fixed point theorem of Reich. Can. Math. Bull. 16, $201-206$ (1973)

7. Subrahmanyam, PV: Completeness and fixed-points. Monatshefte Math. 80, 325-330 (1975)

8. Kannan, R: Some results on fixed points. II. Am. Math. Mon. 76, 405-408 (1969)

9. Rhodes, BE, Sessa, S, Khan, MS, Khan, MD: Some fixed point theorems for Hardy Rogers type mappings. Int. J. Math. Math. Sci. 7(1), 75-87 (1984)

10. Abbas, M, Aydi, H, Radenović, S: Fixed point of Hardy-Rogers contractive mappings in partially ordered partial metric spaces. Int. J. Math. Math. Sci. 2012, Article ID 313675 (2012). doi:10.1155/2012/313675

11. Kumari, PS, Kumar, WV, Sarma, R: New version for Hardy and Rogers type mapping in dislocated metric space. Int. J. Basic Appl. Sci. 1(4), 609-617 (2012)

12. Kirk, WA, Srinavasan, PS, Veeramani, P: Fixed points for mapping satisfying cyclical contractive conditions. Fixed Point Theory 4, 79-89 (2003)

13. Mongkolkeha, C, Kumam, P: Best proximity point theorems for generalized cyclic contractions in ordered metric spaces. J. Optim. Theory Appl. 155, 215-226 (2012)

14. Sintunavarat, W, Kumam, P: Common fixed point theorem for cyclic generalized multi-valued contraction mappings. Appl. Math. Lett. 25(11), 1849-1855 (2012)

15. Nashine, HK, Pathak, RP, Somvanshi, PS, Pantelic, S, Kumam, P: Solutions for a class of nonlinear Volterra integral and integro-differentialequation using cyclic $(\varphi, \psi, \theta)$-contraction. Adv. Differ. Equ. 2013, 106 (2013)

16. Zoto, K, Kumari, PS, Hoxha, E: Some fixed point theorems and cyclic contractions in dislocated and dislocated quasi-metric spaces. Am. J. Numer. Anal. 2(3), 79-84 (2014)

17. Radenović, S: Some remarks on mappings satisfying cyclical contractive conditions. Afr. Math. (2015) doi:10.1007/s13370-015-0339-2

18. Abbas, M, Nazir, T, Gopal, D: Common fixed point results for generalized cyclic contraction mappings. Afr. Math. 26(1), 265-273 (2015)

19. Radenović, S, Dosenović, T, Lampert, TA, Golubović, Z: A note on some recent fixed point results for cyclic contractions in b-metric spaces and an application to integral equations. Appl. Math. Comput. 273, 155-164 (2016)

20. Karpagam, S, Agrawal, S: Best proximity points theorems for cyclic Meir-Keeler contraction maps. Nonlinear Anal. 74, 1040-1046 (2011)

21. Zlatanov, B: Best proximity points for $p$-summing cyclic orbital Meir-Keeler contractions. Nonlinear Anal., Model. Control 20(4), 528-544 (2015)

22. Karapınar, E, Ramaguera, S, Kenan, T: Fixed points for cyclic orbital generalized contractions on complete metric spaces. Cent. Eur. J. Math. 11(3), 552-560 (2013)

23. Wardowski, D: Fixed point theory of a new type of contractive mappings in complete metric spaces. Fixed Point Theory Appl. 2012, Article ID 94 (2012)

24. Shukla, S, Radenović, S: Some common fixed point theorems for F-contraction type mappings in 0-complete partial metric spaces. J. Math. 2013, Article ID 878730 (2013)

25. Minak, G, Helvaci, A, Altun, I: Ćirić type generalized F-contractions on complete metric spaces and fixed point results. Filomat 28(6), 1143-1151 (2014)

26. Kumari, PS, Zoto, K, Panthi, D: $d$-Neighborhood system and generalized F-contraction in dislocated metric space SpringerPlus 4(1), 1-10 (2015)

27. Sedghi, S, Shobe, N, Aliouche, A: A generalization of fixed point theorems in S-metric spaces. Mat. Vesn. 64(3), 258-266 (2012)

28. Azam, A, Fisher, B, Khan, M: Common fixed point theorems in complex valued metric spaces. Numer. Funct. Anal. Optim. 32(3), 243-253 (2011)

29. Abbas, et al.: Generalized coupled common fixed point results in partially ordered A-metric spaces. Fixed Point Theory Appl. 2015, Article ID 64 (2015)

30. Sarma, IR, Rao, JM, Kumari, PS, Panthi, D: Convergence axioms on dislocated symmetric spaces. Abstr. Appl. Anal. 2014, Article ID 745031 (2014). doi:10.1155/2014/7450317

31. Kumari, PS, Ramana, CV, Zoto, K: On quasi-symmetric space. Indian J. Sci. Technol. 7(10), 1583-1587 (2014)

32. Kumari, PS, Sarma, IR, Rao, JM: Metrization theorem for a weaker class of uniformities. Afr. Math. (2015). doi:10.1007/s13370-015-0369-9

33. Kumari, PS, Panthi, D: Cyclic contractions and fixed point theorems on various generating spaces. Fixed Point Theory Appl. 2015, Article ID 153 (2015) 
34. Sarma, IR, Kumari, PS: On dislocated metric spaces. Int. J. Math. Arch. 3(1), 7-27 (2012)

35. Kumari, PS, et al.: Common fixed point theorems on weakly compatible maps on dislocated metric spaces. Math. Sci. 6, $71(2012)$

36. Kumari, PS: On dislocated quasi metrics. J. Adv. Stud. Topol. 3(2), 66-74 (2012)

37. Panthi, D, Jha, K, Jha, PK, Kumari, PS: A common fixed point theorem for two pairs of mappings in dislocated metric space. Am. J. Comput. Math. 5, 106-112 (2015)

38. Kumari, PS, Ramana, CV, Zoto, K, Panthi, D: Fixed point theorems and generalizations of dislocated metric spaces. Indian J. Sci. Technol. 8(S3), 154-158 (2015)

39. Panthi, D: Common fixed point theorems for compatible mapping in dislocated metric space. Int. J. Math. Anal. 9(45), 2235-2242 (2015)

40. Zoto, K, Isufati, A, Kumari, PS: Fixed point results and E.A-property in dislocated and dislocated quasi-metric spaces. Turk. J. Anal. Number Theory 3(1), 24-29 (2015)

Submit your manuscript to a SpringerOpen ${ }^{\circ}$ journal and benefit from:

- Convenient online submission

Rigorous peer review

- Immediate publication on acceptance

- Open access: articles freely available online

- High visibility within the field

- Retaining the copyright to your article 